\title{
Comparative Study between Conservative Breast Surgery and Modified Radical Mastectomy in Triple Negative Cases
}

Eid Rezk El Gammal, Ayman Mohamad Abdulmohaymen, Bashir Adel M. M. Abdulmutaleb*

Surgical Oncology Department, Faculty of Medicine, Al-Azhar University

*Corresponding author: Bashir Adel M. M. Abdulmutaleb ,Mobile: (+20) 1004543591, Email: bashher@gmail.com

\begin{abstract}
Background: Breast cancer has a significant concern in modern researches due its mortality and associated psychological aspects.

Aim of the Work: The aim of this work is to compare conservative breast surgery (CBS) and modified radical mastectomy (MRM) regarding oncologic and cosmetic outcomes in women with primary breast cancer negative for estrogen receptor, progesterone receptor, and human epidermal growth factor receptor 2 triple-negative breast cancer (TNBC).

Patients and Methods: This retrospective study included 20 patients presented by breast cancer. All patients underwent surgery at Al-Azhar University Hospitals. 10 patients were treated by conservative breast surgery and 10 patients were treated by modified radical mastectomy.

Results: We investigated Overall Survival in patients with TNBC treated with mastectomy compared with those receiving CBS. The analysis indicated that patients with CBS had better survival than patients with mastectomy in Overall Survival $(\mathrm{P}<0.001)$.

Conclusion: patients with CBS exhibited improved in TNBC may be associated with the baseline characteristics between two groups. Most patients in the CBS group presented with better survival than did patients in the mastectomy group. CBS was favored over mastectomy.
\end{abstract}

Keywords: Breast cancer, Conservative breast surgery, Modified radical mastectomy.

\section{INTRODUCTION}

Breast cancer is the most common cancer in women throughout the world ${ }^{(\mathbf{1})}$. Breast conserving surgery and radiotherapy have been shown to provide similar local control and survival rates to radical procedures in the surgical treatment of early breast cancer ${ }^{(2)}$. In 1994, lumpectomy with simultaneous bilateral reduction mammoplasty were performed as a solution for cancer breast with macromastia and oncoplastic breast surgery was defined for the first time ${ }^{(3)}$.

Currently, patients with breast cancer are managed using clinical and histologic parameters, such as tumor size, lymph node (LN) status, and grade in conjunction with standardized immunohistochemical assessment of hormone receptors (ie, estrogen receptor [ER], progesterone receptor $[\mathrm{PR}]$ ) and human epidermal growth factor receptor 2 (HER2) testing. Locoregional management of breast cancer has been implemented based on results of randomized controlled trials comparing CBS and MRM ${ }^{(4)}$.

In these studies, locoregional outcome was not investigated with respect to molecular and/or biologic heterogeneity of breast cancer. Indeed, genomic and molecular profiling have paved the way to a paradigm shift toward new molecular classification with at least three major molecular subtypes (5) associated with differences in survival and response to treatment. To approximate these molecular subtypes, most studies are focused on biologic subtyping using ER, PR, and HER2 as biomarkers (6).
In particular, triple-negative breast cancers (TNBCs), which account for approximately $10 \%$ to $17 \%$ of all patients with breast cancer ${ }^{(5)}$, present poorly differentiated tumors lacking expression of ER, PR, and HER2 on immunohistochemical analysis; they are characterized by a high proliferation rate and increased aggressiveness compared with other subtypes ${ }^{(6)}$.

Because endocrine and HER2-targeted therapies cannot be offered, conventional cytotoxic chemotherapy followed by adjuvant RT is the standard of care for patients with TNBC. The paucity of therapeutic options emphasizes the urgent need to optimize the current locoregional management of patients with TNBC and reduce their risk of locoregional recurrence (LRR) ${ }^{(7)}$.

\section{AIM OF THE WORK}

The aim of this study is to compare CBS with MRM regarding oncologic and cosmetic outcomes with primary breast cancer negative for estrogen receptor, progesterone receptor, and human epidermal growth factor receptor 2 (triple-negative breast cancer $[\mathrm{TNBC}])$.

\section{PATIENTS AND METHODS}

Study design: prospective randomized comparative study which was approved by the Ethics Board of Al-Azhar University.

- Study population: breast cancer patients.

- Patient's number: 20 patients.

- Study venue: Al-Azhar University Hospitals. 
Inclusion criteria in the study:

- Patients with proven histopathology of early (stage III) breast cancer.

- Triple negative patients (Estrogen receptor, Progesterone receptor and Human epidermal growth factor receptor 2 HER2).

- No other lesions in the same or contralateral breast.

Exclusion criteria in the study:

- Patient with contraindication of CBS or radiation.

- Medically unfit for surgery.

- Patient with past history of breast cancer.

Patients were included if they agree to be included in the study and an informed consent was obtained.

\section{Methods}

All the twenty patients were subjected to the following:

1-Clinical data for every patient was recorded in a printed sheet:

\section{A-History:}

Personal history: Name, Age, Sex, Occupation, Residence and Special habits.

Present history: Analysis of complaint: Onset, Course. Risk factors.

Past history: Neurological, Cardiac, Operations, Drug intake, Hepatic disease, Lung disease, Similar conditions, Vascular procedure or Allergies.

\section{Family history}

\section{B-Examination:}

I. General examination: Temperature, Respiration, Pulse, Weight, Head and neck, Heart and Abdomen.

Ethical approval:

The study protocol was approved by the Research Ethics Committee of the Faculty of Medicine, AlAzhar University.

\section{Statistical Methods}

All the collected data were coded on the computer and the statistical analysis was done using SPSS program (Statistical Package for Social Science).

\section{RESULTS}

The present study was conducted on 20 patients, 10 patients treated with MRM and 10 patients treated with CBS.

Their age ranged between 41-65 years in CBS group compared with 45-70 years with a mean age shown in (Table 1).

Table 1: Distribution of the studied patients regarding their age

\begin{tabular}{|c|c|c|c|}
\hline & CBS & MRM & p-value \\
\hline $\begin{array}{c}\text { Age } \\
(\text { Mean } \pm \text { S.D })\end{array}$ & $57.2 \pm 12.33$ & $58.2 \pm 11.24$ & $>0.05$ \\
\hline
\end{tabular}

In CBS group $6(60 \%)$ of patients were married compared with 4 (40\%) in MRM group of patients.
There was no statistically significant difference in this distribution.

In CBS group 6 (60\%) of patients were affected on the left side compared with $5(50 \%)$ in MRM group of patients. There was no statistically significant difference in this distribution.

In CBS group tumor size was $<\mathbf{2} \mathbf{~ c m}$ in $6(60 \%)$ of patients w compared with $5(50 \%)$ in MRM group of patients. (2-5) cm in $4(40 \%)$ and $4(40 \%)$ in CBS and MRM groups respectively. One patient in MRM had (> 5) cm tumor size. Statistical correlation was found (p-value: $\mathbf{0 . 0 4 7}$ ).

Table 2: Distribution of the studied patients regarding tumor size.

\begin{tabular}{|l|c|c|c|}
\hline & CBS & MRM & p-value \\
\hline \multicolumn{4}{|c|}{ T-size } \\
\cline { 1 - 3 } T1 (<2) & $6(60 \%)$ & $5(50 \%)$ & \multirow{2}{*}{$\mathbf{0 . 0 4 7}$} \\
\cline { 1 - 3 } T2 (2-5) & $4(40 \%)$ & $4(40 \%)$ & \\
\hline T3 (> 5) & $0(0 \%)$ & $1(10 \%)$ & \\
\cline { 1 - 3 }
\end{tabular}

In CBS group tumour size was $<2 \mathbf{~ c m}$ in $6(60 \%)$ of patients w compared with $5(50 \%)$ in MRM group of patients. (2-5) cm in $4(40 \%)$ and $4(40 \%)$ in CBS and MRM groups respectively. One patient in MRM had (> 5) cm tumour size. This was statistically significant difference (p-value: $\mathbf{0 . 0 4 7}$ ).

Table 3: Distribution of the studied patients regarding tumor stage.

\begin{tabular}{|c|c|c|c|}
\hline & CBS & MRM & p-value \\
\hline \multicolumn{3}{|c|}{ T-stage } \\
\cline { 1 - 3 } Tmic /T1a/T1b & 2 & 2 & \multirow{2}{*}{0.035} \\
\hline T1c & 5 & 6 & \\
\hline T2 & 3 & 2 & \\
\hline
\end{tabular}

In CBS group tumor grade II was found in $3(30 \%)$ of patients compared with $2(20 \%)$ in MRM group of patients. Tumor grade III and IV was present in 7 (70\%) and $8(80 \%)$ in CBS and MRM groups respectively. This was statistically significant difference (p-value: $\mathbf{0 . 0 0 1}$ ).

Table 4: Distribution of the studied patients regarding tumor grade.

\begin{tabular}{|c|c|c|c|}
\hline & CBS & MRM & p-value \\
\hline \multicolumn{3}{|c|}{ Grade } \\
\cline { 1 - 3 } I & 0 & 0 & \multirow{2}{*}{0.001} \\
\cline { 1 - 2 } II & 3 & 2 & \\
\hline III and IV & 7 & 8 & \\
\hline
\end{tabular}

In CBS group, $\mathrm{N} 0$ was presented in $7(70 \%)$ of patients compared with 8 (80 \%) in MRM group of patients. N1 was in $3(20 \%)$ and $2(20 \%)$ in CBS and MRM groups respectively. N2 presented in only one patient in CBS group. This was statistically significant difference (p-value: $\mathbf{0 . 0 2 2}$ ). 
Table 5: Distribution of the studied patients regarding Lymph node involvement.

\begin{tabular}{|l|c|c|c|}
\hline & CBS & MRM & p-value \\
\hline \multicolumn{4}{|c|}{ Lymph node involvement } \\
\hline N0 & 7 & 8 & \multirow{2}{*}{$\mathbf{0 . 0 2 2}$} \\
\cline { 1 - 3 } N1 & 2 & 2 & \\
\hline N2 & 1 & 0 & \\
\hline
\end{tabular}

The hospital stays ranged from 1 to 3 days in CBS group and from 2 days to 4 days in MRM group.

In CBS group Locoregional recurrence occurred in 1 $(10 \%)$ of patients and $1(10 \%)$ in MRM group of patients. Distant metastases occurred in $1(10 \%)$ and $1(10 \%)$ in CBS and MRM groups respectively. Locoregional + Distant metastases occurred in only one patient in CBS group which was statistically nonsignificant (p-value: > 0.05).

Table 6: Distribution of the studied patients regarding relapse.

\begin{tabular}{|c|c|c|c|}
\hline & CBS & MRM & p-value \\
\hline \multicolumn{4}{|l|}{ Relapse } \\
\hline Locoregional recurrence & 1 & 1 & \multirow{3}{*}{$>0.05$} \\
\hline Distant metastases & 1 & 1 & \\
\hline Locoregional + Distant & 1 & 0 & \\
\hline
\end{tabular}

Wound healing time:

In CBS group wound healing time was $6.1 \pm$ 2.34 days compared with $7.4 \pm 3.36$ in MRM group of patients. This was statistically non-significant ( $\mathrm{p}$ value: $>0.05)$.

\section{Wound complications:}

In CBS group seroma formation occurred in 1 $(10 \%)$ of patients and $1(10 \%)$ in MRM group of patients equally. Wound infection occurred in only one patient in MRM group of patients. Considering small sample size, this was statistically nonsignificant (p-value: > 0.05).

Table 2: Distribution of the studied patients regarding Wound complications

\begin{tabular}{|c|c|c|c|}
\hline & CBS & MRM & p-value \\
\hline \multicolumn{3}{|c|}{ Wound } \\
\cline { 1 - 3 } Seroma formation & 1 & 1 & \multirow{2}{*}{$>0.05$} \\
\hline Wound infection & 0 & 1 & \\
\hline
\end{tabular}

\section{Comparison of survival between mastectomy and CBS}

Overall Survival was investigated in patients with TNBC treated with mastectomy compared with those receiving CBS. Kaplan-Meier analysis was used to generate Overall Survival for these two surgical types (Figure ).

The analysis indicated that patients with CBS had better survival than patients with mastectomy in Overall Survival $(\mathrm{P}<0.001)$.

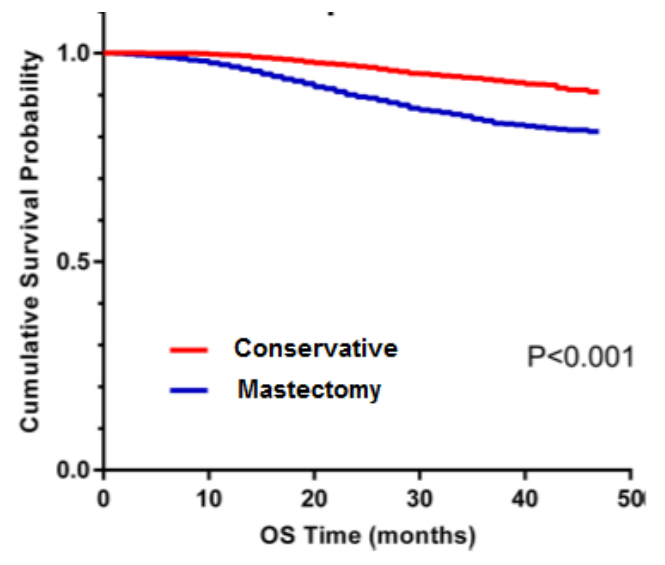

Figure 1: Comparison of survival between mastectomy and CBS

In the Multivariate analysis, excellent survival was identified in the CBS group when compared with the mastectomy group (HR, $0.579 ; 95 \% \mathrm{CI}, 0.488$ to 0.687 ; $\mathrm{P}<0.001$, for Overall Survival).

Table 3: Multivariate Cox proportional hazard regression model of overall survival (OS)

\begin{tabular}{|c|c|c|}
\hline \multirow[t]{2}{*}{ Variables } & \multicolumn{2}{|l|}{ OS } \\
\hline & HRs $(95 \%$ CI) & $P c$ \\
\hline \multicolumn{3}{|c|}{ Marital status } \\
\hline Married & Reference & Reference \\
\hline Not Married & $1.308(1.129-1.516)$ & $<0.001$ \\
\hline \multicolumn{3}{|l|}{ Grade } \\
\hline I & $0.263(0.085-0.820)$ & 0.021 \\
\hline II & $0.879(0.707-1.093)$ & 0.246 \\
\hline III and IV & Reference & Reference \\
\hline \multicolumn{3}{|c|}{ Lymph node involvement } \\
\hline No & Reference & Reference \\
\hline N1 & $1.902(1.540-2.349)$ & $<0.001$ \\
\hline N2 & $3.858(2.527-5.889)$ & $<0.001$ \\
\hline \multicolumn{3}{|c|}{ Tumor Size $(\mathbf{c m})$} \\
\hline T1 $(<2)$ & Reference & Reference \\
\hline $\mathrm{T} 2(2-5)$ & $1.534(1.163-2.022)$ & 0.002 \\
\hline T3 (> 5) & $2.862(2.069-3.958)$ & $<0.001$ \\
\hline
\end{tabular}

\section{DISCUSSION}

According to SEER data to examine the different outcomes between CBS+RT and mastectomy for TNBC patients. The present study, showed that CBS+RT could improve B CSS and OS compared with mastectomy. Furthermore, upon stratifying the TNBC patients according to age, histology grade, stage, tumor size, and LN status, most patients with CBS+ radiotherapy (RT) presented with better survival than did patients with mastectomy, except for the grade I and stage I patients, who had the same survival in the CBS+RT and mastectomy groups. These findings indicated that $\mathrm{CBS}+\mathrm{RT}$ is at least equivalent to mastectomy in terms of BCSS and OS. The finding that the longterm survival of early-stage breast cancer patients 
treated with $\mathrm{CBS}+\mathrm{RT}$ is at least equivalent to treatment with mastectomy has been demonstrated in several prospective and retrospective randomized controlled trials ${ }^{(7,8,9)}$.

Recently, a Dutch population-based study conducted a comparison of 10-year OS and breast relative survival between $\mathrm{CBS}+\mathrm{RT}$ and mastectomy for patients with early breast cancer (T1-2, N0-1, M0), which further confirmed the availability of $\mathrm{CBS}+\mathrm{RT}^{\left({ }^{(10)}\right.}$. However, these studies did not analyze the different outcomes between CBS+RT and mastectomy for TNBC patients. Furthermore, it was not observed that T1-2N0 TNBC treated with mastectomy without RT exhibited a significant increased risk of LRR compared with those treated with CBS until 2011 in a study from a cancer registry at a single institution ${ }^{(11)}$.

Additionally, most studies on locoregional treatment of TNBC patients have been limited by relatively small sample sizes and have demonstrated inconsistent outcomes. Nguyen $\boldsymbol{e t}$ al. identified a total of 1325 patients with TNBC who underwent CBS or mastectomy and found that the five-year LRR-free survival and distant metastasis-free survival rates were higher in the CBS group ${ }^{(12)}$. A cohort study including 1,138 Asian TNBC patients who were treated with CBS, mastectomy alone or mastectomy plus RT showed that for 775 T1-2N0$1 \mathrm{M} 0$ TNBC patients, the adjusted risks of mortality in the three groups were not significantly different (13). However, their study consisted of 11,514 TNBC patients, constituting a larger cohort and a wide range of patients from the SEER database, and provided more convincing evidence that $\mathrm{CBS}+\mathrm{RT}$ may not be contraindicated for TNBC patients. Additionally, the primary outcomes of BCSS and OS can represent the ultimate effects of different surgical types ${ }^{(14)}$.

Our results showed that patients with CBS+RT exhibited improved OS in TNBC that may be associated with the baseline characteristics between the two groups and the application of RT in the CBS group. Considering baseline characteristics, we stratified the whole patient population according to age, grade, and $\mathrm{T}$, and $\mathrm{N}$ stages, and most patients in the CBS+RT group presented with better survival than did patients in the mastectomy group, except for the grade I and stage I patients. Furthermore, we observed that $69.6 \%$ of patients underwent mastectomy without RT in our study. Thus, we suspected that the CBS+RT was favored over mastectomy may due to RT (15).

Accumulating evidence shows that radiation can induce an abscopal effect by stimulating the immune system to inhibit distant metastasis lesions. Additionally, we recognized that the BRCA1 mutation in TNBC patients might influence our results. A relevant study indicated that tumors lacking functional BRCA1 were highly radiosensitive (12). Therefore, for TNBC patients, who share a considerable overlap in BRCA1 mutation, in the context of CBS + RT to the breast and surrounding tissue could eradicate recessive BRCA1- deficient tumor lesions and thereby decrease LRR (16). However, to date, with no consistent evidence available, the significance of RT for CBS requires further exploration with large-scale prospective studies ${ }^{(17)}$.

In the present work, there were 164 cases of 1003 tumors larger than $5 \mathrm{~cm}$ in size among TNBC patients accepting CBS+RT; those patients showed superior survival compared to those in the mastectomy group. This finding seemed discordant with the National Comprehensive Cancer Network $(\mathrm{NCCN})$ guidelines that tumors larger than $5 \mathrm{~cm}$ in size are at high risk of recurrence for patients with CBS+RT. However, over the past several decades, neoadjuvant therapy (NAT) has proven beneficial for locally advanced breast cancer, as it renders inoperable tumors operable or downstages them, thus increasing the rates of CBS. In a large national database of 5,685 patients with T3 primary tumors, $15.6 \%$ of whom received CBS, similar survival rates were found for CBS and mastectomy. Furthermore, a study suggested that CBS with RT was significantly associated with a lower mortality risk than was mastectomy without RT for 363 T3-4, N2-3, M0 TNBC patients. Therefore, we speculated that CBS+RT could also be available after NAT in advanced TNBC patients, although information on NAT was absent from our study ${ }^{(\mathbf{1 8})}$.

One of the strengths of the present study rests on the sizable number of triple-negative breast cancer patients in the SEER database, which ensures the strength and objectivity of our conclusions. Inevitably, our study had several limitations. In terms of follow-up data, it is a well-known fact that information regarding Her-2 expression in the SEER database was not available until 2010. Therefore, we were compelled to focus on the short-term survival outcomes after initial diagnosis and to identify any outcome-related factors; in this context, an inadequate follow-up duration may lead to skewed results. However, concerning TNBC subtype, the early peaks of recurrence and mortality occur within the first 2-3 years after diagnosis. Additionally, information on adjuvant or neoadjuvant chemotherapy not available for our study and probably unknown variables of tumor biology that we are still not aware of may exert a certain influence on our results ${ }^{(\mathbf{1 9})}$.

In conclusion, from our study on SEER data, CBS+RT displayed elevated OS in TNBC patients compared to mastectomy, at least equally. Although cosmetic impairments resulting from mastectomy can 
be addressed with immediate reconstruction, we still should consider the benefits of improved outcomes and an avoidable deterioration in quality life during the surgical decision-making process. Therefore, $\mathrm{CBS}+\mathrm{RT}$ is a preferable choice for TNBC patients if given adequate adjuvant treatment ${ }^{(\mathbf{1 8})}$.

\section{CONCLUSION}

Patients with CBS exhibited improved in TNBC which may be associated with the baseline characteristics between two groups. Most patients in the CBS group presented with better survival than did patients in the mastectomy group. CBS was favored over mastectomy.

\section{REFERENCES}

1. Albert JM, Gonzalez-Angulo AM, Guray $\mathrm{M}$ et al. (2010): Estrogen/progesterone receptor negativity and HER2 positivity predict locoregional recurrence in patients with T1a, bN0 breast cancer. Int J Radiate Oncol Biol Phys., 77:1296-1302.

2. Bauer KR, Brown M, Cress RD et al. (2007): Descriptive analysis of estrogen receptor (ER)-negative, progesterone receptor (PR)-negative, and HER2negative invasive breast cancer, the so-called triplenegative phenotype: A population-based study from the California cancer Registry. Cancer, 109:1721-1728

3. Carey LA, Perou CM, Livasy CA et al. (2006): Race, breast cancer subtypes, and survival in the Carolina Breast Cancer Study. JAMA., 295:2492-2502.

4. Carey LA, Dees EC, Sawyer L et al. (2007): The triple negative paradox: Primary tumor chemosensitivity of breast cancer subtypes. Clin Cancer Res., 13:23292334.

5. Clarke M, Collins R, Darby S et al. (2005): Effects of radiotherapy and of differences in the extent of surgery for early breast cancer on local recurrence and 15-year survival: An overview of the randomized trials. Lancet, 366:2087-2106.

6. Dent R, Trudeau M, Pritchard KI et al. (2007): Triple-negative breast cancer: Clinical features and patterns of recurrence. Clin Cancer Res., 13:4429-4434.

7. Fisher B, Anderson S, Bryant J et al. (2002): Twentyyear follow-up of a randomized trial comparing total mastectomy, lumpectomy, and lumpectomy plus irradiation for the treatment of invasive breast cancer. N Engl J Med., 347:1233-1241.

8. Freedman GM, Anderson PR, Li T et al. (2009): Locoregional recurrence of triple-negative breast cancer after breast-conserving surgery and radiation. Cancer, 115:946-951.

9. Gabos Z, Thoms J, Ghosh S et al. (2010): The association between biological subtype and locoregional recurrence in newly diagnosed breast cancer. Breast Cancer Res Treat., 124:187-194.

10. Haffty BG, Yang $Q$, Reiss $M$ et al. (2006): Locoregional relapse and distant metastasis in conservatively managed triple negative early-stage breast cancer. J Clin Oncol., 24:5652-5657.

11. Kyndi M, Sørensen FB, Knudsen H et al. (2008): Estrogen receptor, progesterone receptor, HER-2, and response to postmastectomy radiotherapy in high-risk breast cancer: The Danish Breast Cancer Cooperative Group. J Clin Oncol., 26:1419-1426.

12. Nguyen PL, Taghian AG, Katz MS et al. (2008): Breast cancer subtype approximated by estrogen receptor, progesterone receptor, and HER-2 is associated with local and distant recurrence after breast-conserving therapy. J Clin Oncol., 26:23732378.

13. Nishimura R, Arima $\mathbf{N}$ (2008): Is triple negative a prognostic factor in breast cancer? Breast Cancer, 15:303-308.

14. Perou CM, Sørlie T, Eisen MB et al. (2000): Molecular portraits of human breast tumors. Nature, 406:747-752.

15. Sørlie T, Perou CM, Tibshirani R et al. (2001): Gene expression patterns of breast carcinomas distinguish tumor subclasses with clinical implications. Proc Natl Acad Sci USA., 98:1086910874.

16. Tenofsky PL, Dowell P, Topalovski T et al. (2014): Surgical, oncologic, and cosmetic differences between oncoplastic and no oncoplastic breast conserving surgery in breast cancer patients. Am J Surg., 2007:398-402.

17. Veronesi U, Cascinelli N, Mariani L et al. (2002): Twenty-year follow-up of a randomized study comparing breast-conserving surgery with radical mastectomy for early breast cancer. N Engl J Med., 347:1227-1232.

18. Voduc KD, Cheang MC, Tyldesley S et al. (2010): Breast cancer subtypes and the risk of local and regional relapse. J Clin Oncol., 28:1684-1691.

19. Yang JD, Lee JW, Cho YK et al. (2012): Surgical techniques for personalized oncoplastic surgery in breast cancer patients with small-to moderate-sized breasts (part 2): volume replacement. J Breast Cancer, $1: 1-6$. 